\title{
Status of XMASS experiment
}

\author{
Ko Abe $\mathrm{Ab}^{\mathrm{ab}}$ \\ Kamioka Observatory, Institute for Cosmic Ray Research, University of Tokyo \\ Higashi-Mozumi 456, Kamioka-cyo, Hida-city, Gifu 506-1205, Japan \\ E-mail: abe@suketto.icrr.u-tokyo.ac.jp
}

XMASS is multi purpose low background and low energy threshold experiment using large amount of liquid xenon. The first stage of XMASS, 800kg detector's main target is Dark Matter search. Total amount of liquid xenon used in $800 \mathrm{~kg}$ detector is about $850 \mathrm{~kg}$ for active volume. With $10^{-4} / \mathrm{day} / \mathrm{keV} / \mathrm{kg}$ background level, the sensitivity of WIMP-nucleon cross section for the spin independent case will be $10^{-45} \mathrm{~cm}^{2}$ at $100 \mathrm{GeV} / c^{2}$ WIMP mass for 5 years exposure.

Liquid xenon has a large scintillation photon yield of 46,000 photons $/ \mathrm{MeV}$, which is as good as $\mathrm{NaI}(\mathrm{Tl})$ scintillator. This enables us to achieve the detection of small energy signals. The key idea of the background reduction in XMASS is self-shielding, to use detector liquid xenon itself as a shield to reduce gamma rays background. Due to its high atomic number $(Z=54)$ and high density $\left(3 \mathrm{~g} / \mathrm{cm}^{3}\right)$ liquid xenon has strong power to shield gamma rays. Using the outer region of liquid xenon as shield, extremely low background environment can be achieved in the detector center region.

In addition to self-shielding, to keep background level as low as $10^{-4} / \mathrm{day} / \mathrm{keV} / \mathrm{kg}$ level, XMASS detector has many key components such as newly developed extremely low BG PMT, water shield, purification system and distillation tower.

The construction of XMASS 800kg detector was completed in fall, 2010 and the water shield is ready to be used for the active veto for cosmic-rays. After the commissioning run, the data taking will be started in late 2010 .

35th International Conference of High Energy Physics (ICHEP2010)

Paris,France

July 22-28, 2010

Speaker

for XMASS collaboration 


\section{XMASS experiment.}

XMASS [1] 800kg detector was installed in the new experimental hall inside Kamioka mine, Japan. Fig. 1 shows a schematic view of $800 \mathrm{~kg}$ detector. OFHC copper PMT holder keeps the 642 hexagonal photomultiplier tubes (PMTs), R10789 Hamamatsu. PMTs are arranged in an approximately spherical shape with an average radius of $40 \mathrm{~cm}$. PMTs photo-cathode cover $62.4 \%$ inner surface of the detector.

The main component of radioactive background is expected to come from the PMTs. The activity level of the PMT is $1 / 10$ of R8778, $1.51 \mathrm{mBq} / \mathrm{PMT}$ for uranium-chain, $0.70 \mathrm{mBq} / \mathrm{PMT}$ for thorium-chain, $<5.1 \mathrm{mBq} / \mathrm{PMT}$ for ${ }^{40} \mathrm{~K}$ and $2.92 \mathrm{mBq} / \mathrm{PMT}$ for ${ }^{60} \mathrm{Co}$. Estimated background rate from the PMTs is less than $10^{-4}$ count $/ \mathrm{day} / \mathrm{kg} / \mathrm{keV}$ inside the $100 \mathrm{~kg}$ fiducial volume.

The purification of xenon in gas phase is performed by SAES getter at the 30L/min of flow rate during the filling with two 200W pulse tube refrigerators. The recirculation of xenon in liquid phase is also possible at a few $\mathrm{L} / \mathrm{min}$ of flow rate for the purification, if necessary. Filter to reduce radon will be installed to the purification system. The distillation tower for $\mathrm{Kr}$ [2] was built to achieve $<1 \mathrm{ppt}$ with about $4 \mathrm{~m}$ length of the tower column and about 1.2 ton of xenon gas was processed in September 2010 for 10 days.

The construction of XMASS 800kg detector was completed in fall, 2010 and the water shield is ready to be used for the active veto for cosmic-rays. After the commissioning run, the data taking will be started in late 2010 .
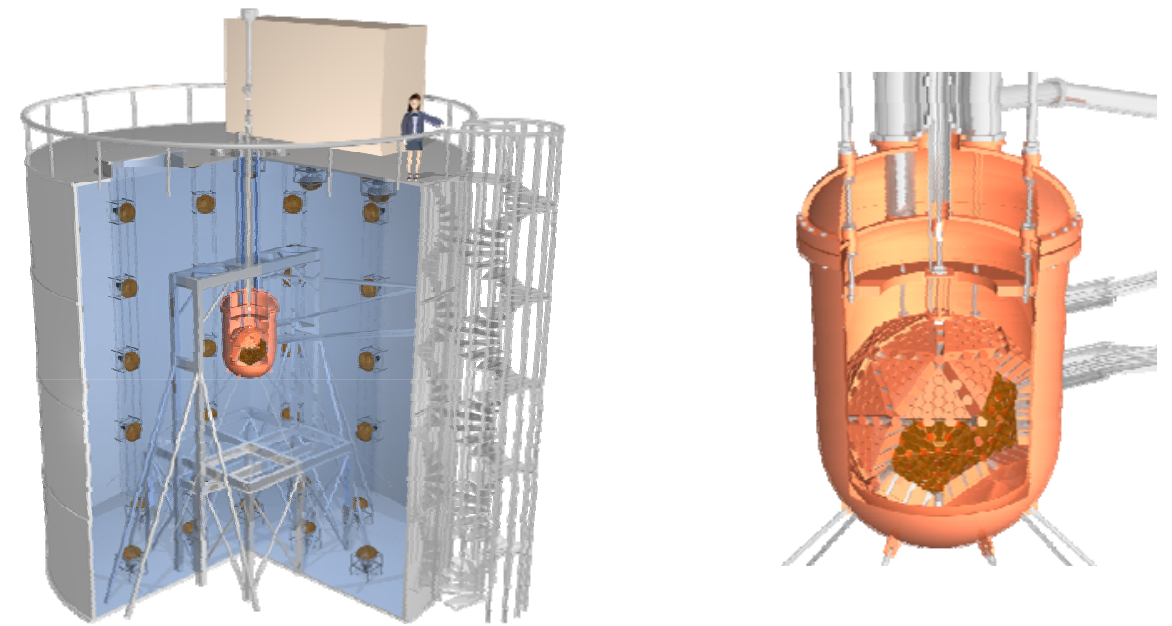

FIGURE 1. Schematic view of XMASS detector. $10 \mathrm{~m}$ diameter and $10 \mathrm{~m}$ height water tank is used for the radiation shield. 7220 inch PMTs are attached on the inner surface of tank and work as active veto counter for the cosmic rays. Two OFHC copper vessel chambers cover the detector to hold liquid xenon with vacuum insulation.

\section{References}

[1] Y. Suzuki et al, Low Energy Solar Neutrino Detection by using Liquid Xenon, [hep$\mathrm{ph} / 0008296]$

[2] K. Abe, Distillation of liquid Xenon to remove Krypton, Astroparticle Physics 31 (2009) 290. 